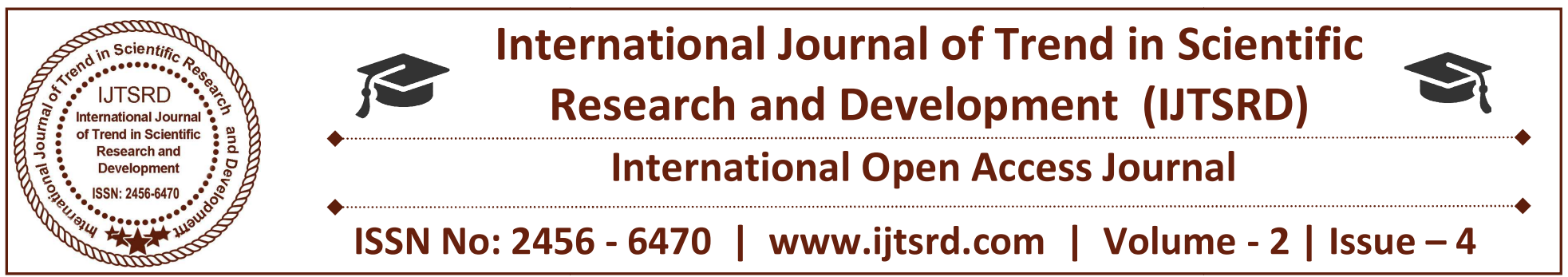

\title{
Guidelines for Approching Steps of Learning Programmable Logic Controllers in Automation
}

\author{
Dr. Wai Phyo Aung \\ Department of Electronic Engineering Technological University Loikaw, Kayah State, Myanmar
}

\begin{abstract}
This paper provides both Theory and Practical of Programmable logic controller (PLC) in Automation engineering. According to various manufacturing company, the nature and useful area of PLC may be different and this paper mainly supports step by step approaching methods to be familiar with what is PLC and how PLC work. Automation purpose is the main application of PLC and the programmers or control supervisors will be able to learn easily with the four levels or steps that perform in this paper.
\end{abstract}

Keywords: PLC, Automation, Methods, levels

\section{INTRODUCTION}

Technological advances in recent years have resulted in the development of the programmable logic controller and a consequential revolution of control engineering. The purpose of a PLC was to directly replace electro-mechanical relays as logic elements, substituting instead a solid-state digital computer with a stored program, able to emulate the interconnection of many relays to perform certain logical tasks. A Programmable Logic Controller, PLC or Programmable Controller is a digital computer used for automation of electromechanical processes. Figure 1 shows the basic arrangement of a PLC system. There are two common types of mechanical design for PLC systems; a single box, and the modular/rack types.

PLC manufacturers have programming software for their PLCs. For example, Mitsubishi have MELSOFT. Their GX Developer supports all MELSEC controllers from the compact PLCs of the MELSEC FX series to the modular PLCs including MELSEC System Q and uses a Windows based environment.
Siemens have SIMATIC STEP 7. This fully complies with the international standard IEC 61131-3 for PLC programming languages. With STEP 7, programmers can select between different programming languages. Practical application of PLC based Industrial Control or Automation system needs fundamental to advance knowledge of computer system, communication or networking and both hardware and software technology of sensors and input/output devices. This paper develops learning approaches of PLC with level1 to level4 by the experiences and teaching environments of author.

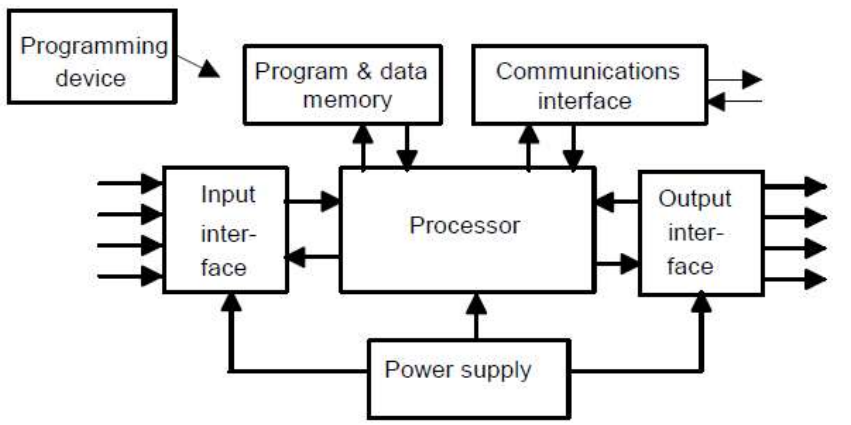

Figure-1 PLC system

\section{II.FOUR LEVELS OF PLC TECHNOLGY}

The user or engineer need to study technological specification of a PLC according to it manufacturing company even higher in the knowledge of PLC. By this point of view, this paper carry out the approaching steps of learning PLCs using OMRON and SEIMENS type. The proposed four levels of learning PLC are as follows:

1. Level1: Simulated monitoring of PLC projects.

2. Level2:Approch PLC projects by Simulated monitoring and Hardware training Kit with low number of $\mathrm{I} / \mathrm{O}$. 
3. Level3: Approch PLC projects by both hardware and software, and also real-time networking for automation.

4. Level4: PLC and HMI programming practices.

\section{A. Software for PLCs}

There are various software for PLC programming and compiling to PLCs.CX-Programmer is software for easy used from OMRON. The easier or user friendly the type, the less input and outputs or performance of PLC can be used. The consideration software of level 1 and level 2 approaches in this paper is CXprogrammer which is user friendly but cannot used with HMI. The CX-programmer window is shown in figure-2.

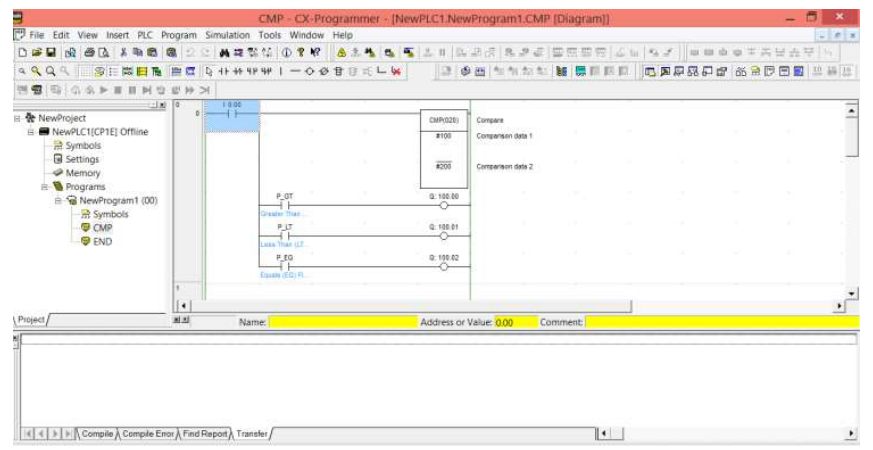

Figure-2 CX Programmer window

The other software is TIA portal which is supports by SEIMENS for S7-1200 and S7-1500 PLCs. TIA stands for Totally Integrated Automation. This software is suitable with the Rack type PLCs and S71200/S7-1500 PLCs are Rack types. The TIA portal window with connecting of PLC to PC process is shown in figure-3.

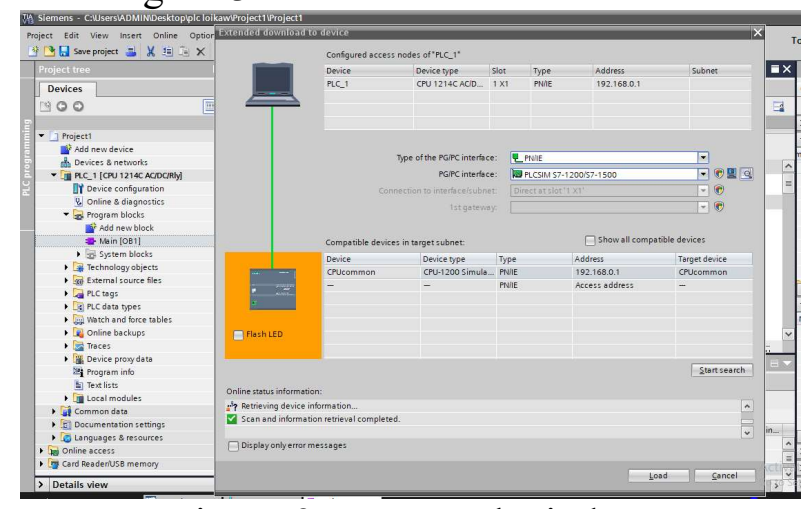

Figure-3 TIA Portal window

\section{B. Types of PLCs}

For OMRON PLCs, CP type is the compact-able or sometimes may called micro type PLCs. The various type of OMRON PLCs according to their performance levels are shown in figure-4.

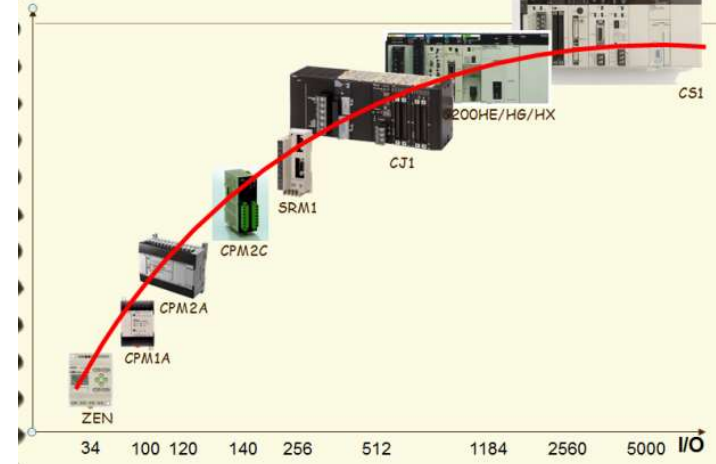

Figure-4 OMRON PLCs according to I/O numbers

SEIMENS PLC types also changes depend on their software version and hardware update. S7-200 and S7-300 are the old version of SEIMENS PLCs. Updated version means update firmware, update CPU with higher performance, larger memory space, higher technology of Ethernet, so on. Figure-5 shows the getting started process of TIA portal and its properties of connectable circumstances.

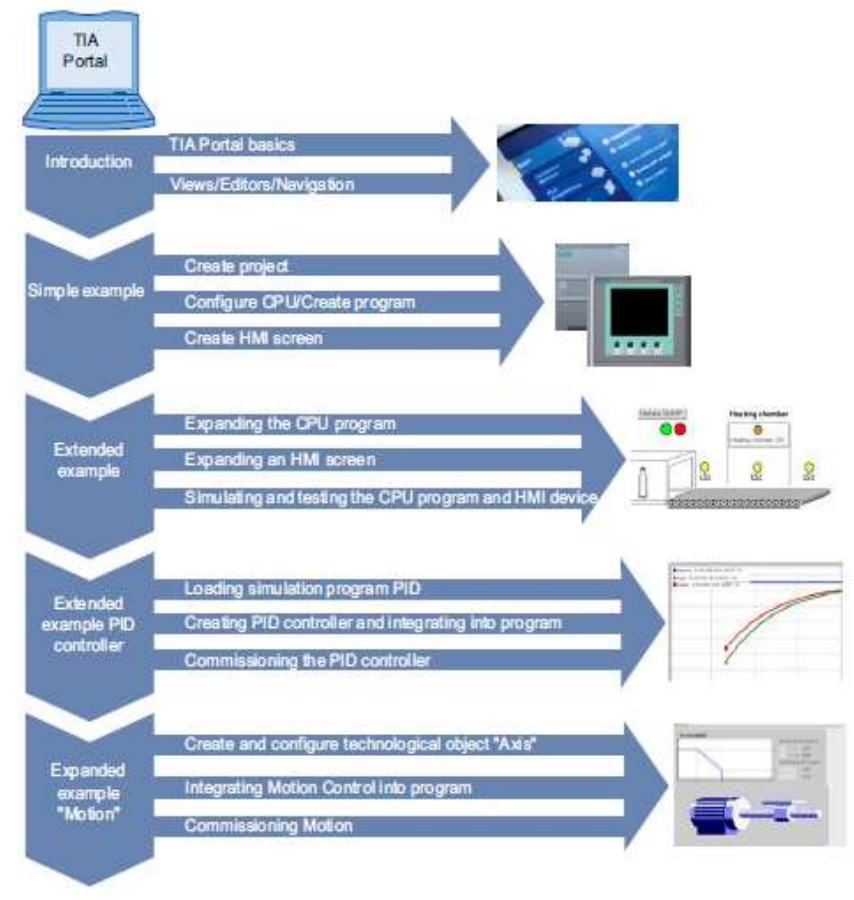

Figure-5 TIA portal getting started

\section{OBJECTIVES OF EACH LEVEL}

By separating four level of learning technologies of PLC automation, the application purposes is different. For example, Engineer cannot be able to design the program or cannot be able to communicate networking between PLC, PC and I/O Machines for PLC programming that interfacing with HMI. The reason is we need to simulate and test our program in simulation and monitoring software tools. So, step by step learning approach can support the technologist more friendly with PLC nature. 


\section{A. Outcomes of Level 1 Approach}

In this level, CX-programmer software only without HMI simulation is to be used. The desire outcomes of level 1 approach which called Simulated monitoring of PLC are as follows:

1. to apply the theory background knowledge of PLCs.

2. to be familiar with ladder diagram(LD), Sequential Flow Text(SFT),Instruction List(IL) programming in software.

3. to practices various sample applications program without hardware problems.

\section{B. Outcomes of Level 2 Approach}

In this level, CP1E OMRON PLC with USB port and CX-Programmer software is to be used. The desire outcomes of level 2 approach which called Simulated monitoring and Hardware training Kit with low number of $\mathrm{I} / \mathrm{O}$ are as follows:

1. to apply the Sourcing or Sinking wiring of input to PLC/ PLC to output.

2. to be familiar with input ports and output ports of PLC but not with relays, motors, encoders, etc.

3. to verify and practices various sample applications program with PLC hardware but not with realtime machines.

\section{Outcomes of Level 3 and Level 4}

In these levels, S7-1200/1500 Seimens PLC with Profinet and TIA portal software is to be used as realtime practice. The desire outcomes of level 3 approach which called both hardware and software, and also real-time networking for automation are as follows:

1. to verify and practices various sample applications program with PLC Software and then with realtime I/Os.

2. to be familiar with real-time machine control for automation purposes.

3. to practices PLC to/from PC and I/O networking.

Level 4 gives one more outcome apart from level 3 that is HMI programming. So, the future higher approach is SCADA (Supervisory Control and Data Acquisition).

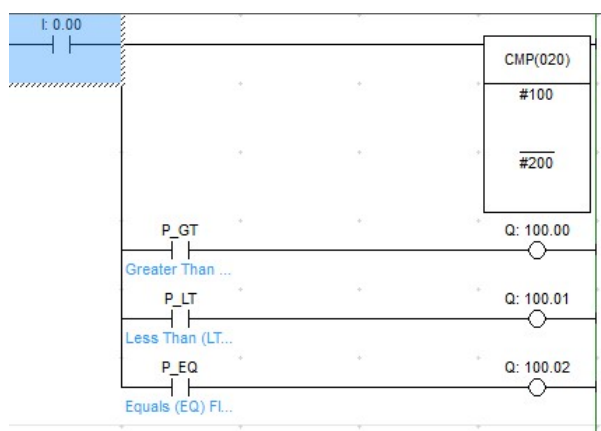

Figure-6 LD program in CX-Programmer

\section{TEST, RESULTS AND CONCLUSION}

Depend on training or teaching environments and candidates, more than five projects of each level can be done for learning PLC. This paper carried out from various projects and some sample results will be shown from figure- 6 to figure-10.Each project can be successfully perform with the following detail flows of study:

1. Process description of desire project.

2. Flow chart.

3. Inputs and Outputs assigning.

4. Ladder Diagram representation

5. Software simulation, I/O testing, real-time.

\begin{tabular}{|c|c|c|c|}
\hline Rung & Instruction & Operand & Comment \\
\hline \multirow[t]{4}{*}{0} & @LD & I: 0.00 & Start input \\
\hline & "ÖR" & "Q: & \\
\hline & ANDNOT & T002 & \\
\hline & OUT & Q: 100.00 & \\
\hline \multirow[t]{3}{*}{1} & LD & Q: 100.00 & \\
\hline & TIM & 000 & timer1 \\
\hline & & \#200 & \\
\hline \multirow[t]{5}{*}{2} & LD & I: 0.01 & \\
\hline & AND & Q: 100.00 & \\
\hline & LD & T002 & \\
\hline & CNT & 000 & Counter1 \\
\hline & & \#3 & \\
\hline \multirow[t]{3}{*}{3} & LD & $\mathrm{C} 000$ & Counter1 \\
\hline & AND & T000 & timer1 \\
\hline & OUT & Q: 100.01 & OK \\
\hline \multirow[t]{3}{*}{4} & LD & Q: 100.01 & OK \\
\hline & TIM & 002 & \\
\hline & & $\# 100$ & \\
\hline
\end{tabular}

Figure-7 timer and counter mnemonic

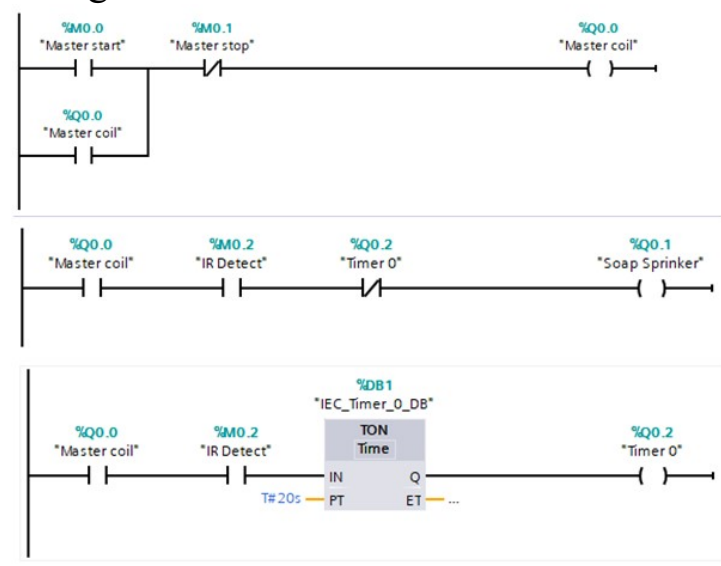



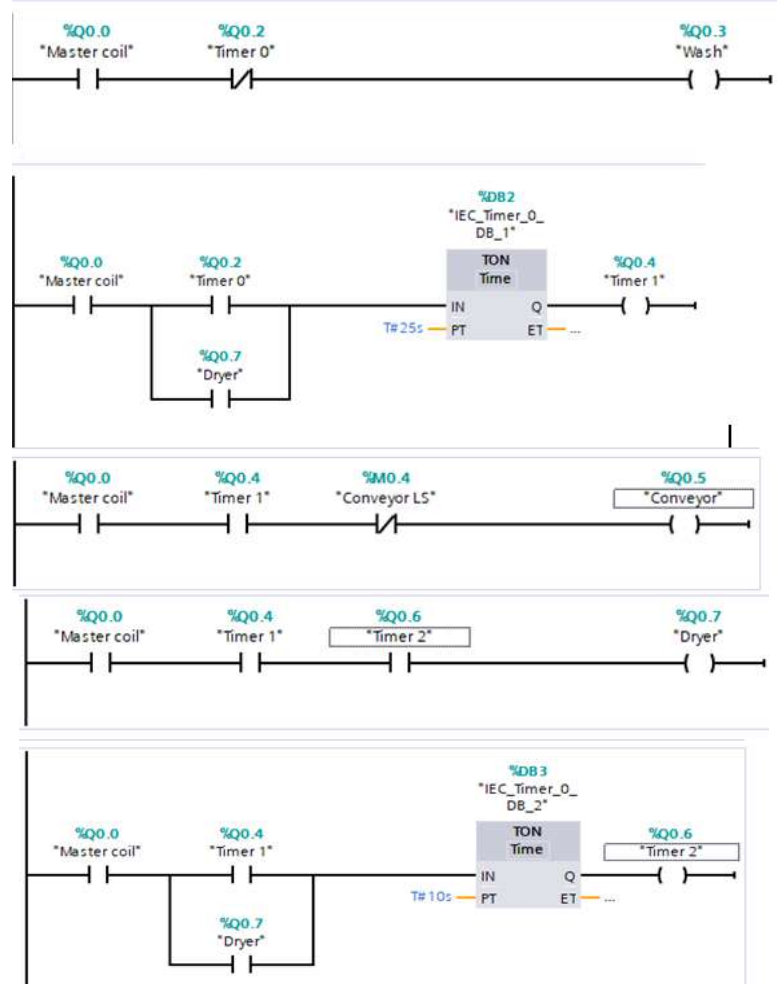

Figure-8 Car washing project in TIA portal

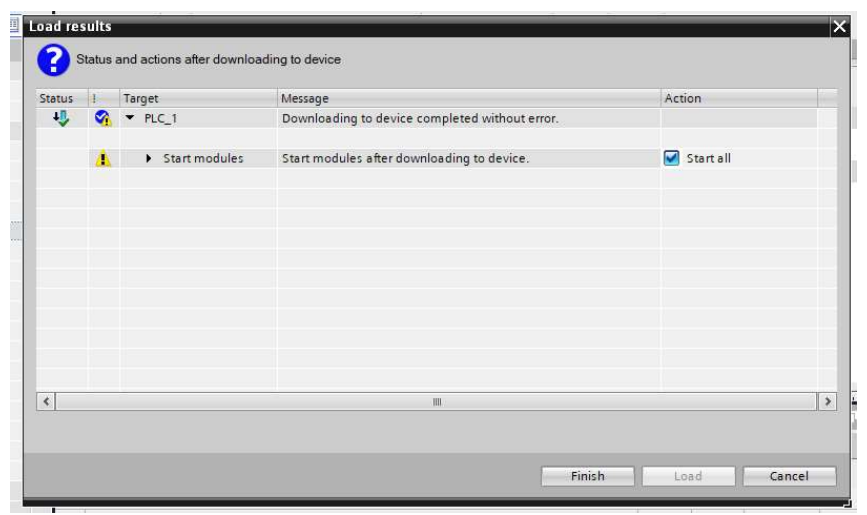

Figure-9 Downloading program to PLC

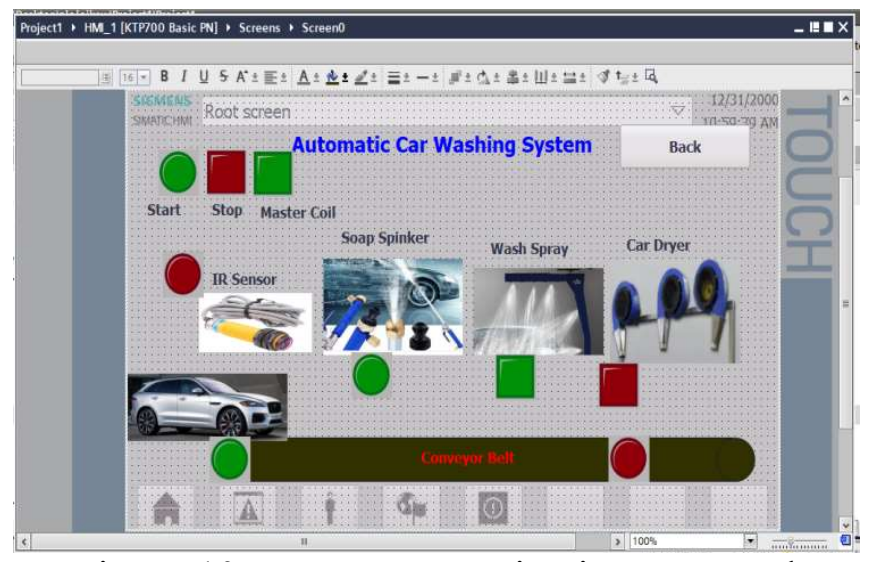

Figure-10 HMI programming in TIA portal

For automation process, when using a PLC, networking is one of important technology that the user should be familiar. As PLCs are the improvement of relay logic, AC and DC relay are used. For example, the specification of $12 \mathrm{AC} / \mathrm{DC} / \mathrm{RLY}$ in a S71200 Seimens PLC means totally 12 inputs and outputs, inputs are DC and output are relay type. The real-time PLC applications are very sensitive in network communication, so, we need to precaution some facts when testing a project.

This paper, now, mainly supports the guidelines of how we can approach to be fulfilled in doing PLC based Automation projects, specially in steps by steps.

\section{ACKNOWLEDGMENT}

Special Thanks are due to Dr. Nyan Phyo Aung, Associate Professor, EcE Dept, TU (Mandalay), for his short training course upon Seimens PLC. The author would like to mention his thanks to all his partners from EcE Dept, TU (Loikaw) for their supports.

\section{REFERENCES}

1. @OMRON coporation 2008-2013 All right reserved, "CX-Programmer Introduction Guide".http://www.ia.omron.com.

2. SEIMENS SIMATIC TIA PORTAL STEP 7 Basic V10.5206 Getting Started, 12/2009, A5E02651459-01. http://www.seimens.com

3. W.Bolton, "Progrmmable Logic Controller, $4^{\text {th }}$ Edition,Copyright@ 2006, W. Bolton. Published by Elsevier Newnes. All rights reserved. ISBN-13: 978-0-7506-8112-4,ISBN-10: 0-7506-8112-8.

4. Prepared by-Eng.Wael Younis, "PLC Lab Manual", http://www.infoPLC.net.

5. Avvaru Ravi Kiran, B.Venkat Sundeep, Ch. Sree Vardhan, Neel Mathews, "The Principle of Programmable Logic Controller and its role in Automation". International Journal of Engineering Trends and Technology- Volume4Issue3- 2013.

6. Vivek Kumar Yadav,Suryansh Tyagi, Gulshan Kumar, Nishant Kumar,Swapril Namekar, "Automatic Car Washing Using PLC", International Journal of Engineering Science and Computing,May 2016.Volume 6 Issue No.5. ISSN 23213361.

7. VVS Madhuri, Naveen Yeerathota, "Automatic Washing Machine Using PLC" , Internaltion Journal of Latest Trends in Engineering and Technology. Volume 7 issue 2 July 2016, ISSN 2278-621X. 